\section{Evolocumab in Pediatric Heterozygous Familial Hypercholesterolemia}

\author{
Raul D. Santos, M.D., Ph.D., Andrea Ruzza, M.D., Ph.D., \\ G. Kees Hovingh, M.D., Ph.D., Albert Wiegman, M.D., Ph.D., François Mach, M.D., \\ Christopher E. Kurtz, M.D., Andrew Hamer, M.D., Ian Bridges, M.Sc., \\ Andrea Bartuli, M.D., Jean Bergeron, M.D., Tamás Szamosi, M.D., \\ Saikat Santra, M.B., B.Chir., Claudia Stefanutti, M.D., Ph.D., \\ Olivier S. Descamps, M.D., Ph.D., Susanne Greber-Platzer, M.D., \\ Ilse Luirink, M.D., Ph.D., John J.P. Kastelein, M.D., Ph.D., \\ and Daniel Gaudet, M.D., Ph.D., for the HAUSER-RCT Investigators*
}

\section{BACKGROUND}

Evolocumab, a fully human monoclonal antibody directed against proprotein convertase subtilisin-kexin type 9, is widely used in adult patients to lower low-density lipoprotein (LDL) cholesterol levels. Its effects in pediatric patients with heterozygous familial hypercholesterolemia are not known.

\section{METHODS}

We conducted a 24-week, randomized, double-blind, placebo-controlled trial to evaluate the efficacy and safety of evolocumab in pediatric patients with heterozygous familial hypercholesterolemia. Patients 10 to 17 years of age who had received stable lipid-lowering treatment for at least 4 weeks before screening and who had an LDL cholesterol level of $130 \mathrm{mg}$ per deciliter $(3.4 \mathrm{mmol}$ per liter) or more and a triglyceride level of $400 \mathrm{mg}$ per deciliter $(4.5 \mathrm{mmol}$ per liter) or less were randomly assigned in a 2:1 ratio to receive monthly subcutaneous injections of evolocumab (420 mg) or placebo. The primary end point was the percent change in LDL cholesterol level from baseline to week 24; key secondary end points were the mean percent change in LDL cholesterol level from baseline to weeks 22 and 24 and the absolute change in LDL cholesterol level from baseline to week 24 .

\section{RESULTS}

A total of 157 patients underwent randomization and received evolocumab (104 patients) or placebo (53 patients). At week 24 , the mean percent change from baseline in LDL cholesterol level was $-44.5 \%$ in the evolocumab group and $-6.2 \%$ in the placebo group, for a difference of -38.3 percentage points $(\mathrm{P}<0.001)$. The absolute change in the LDL cholesterol level was $-77.5 \mathrm{mg}$ per deciliter $(-2.0 \mathrm{mmol}$ per liter $)$ in the evolocumab group and $-9.0 \mathrm{mg}$ per deciliter $(-0.2 \mathrm{mmol}$ per liter $)$ in the placebo group, for a difference of $-68.6 \mathrm{mg}$ per deciliter $(-1.8 \mathrm{mmol}$ per liter $)(\mathrm{P}<0.001)$. Results for all secondary lipid variables were significantly better with evolocumab than with placebo. The incidence of adverse events that occurred during the treatment period was similar in the evolocumab and placebo groups.

\section{CONCLUSIONS}

In this trial involving pediatric patients with familial hypercholesterolemia, evolocumab reduced the LDL cholesterol level and other lipid variables. (Funded by Amgen; HAUSER-RCT ClinicalTrials.gov number, NCT02392559.)
The authors' affiliations are listed in the Appendix. Address reprint requests to Dr. Gaudet at the Department of Medicine, Université de Montréal and ECOGENE-21 Clinical Research Center, 930 JacquesCartier, Chicoutimi, QC G7H 7K9, Canada, or at daniel.gaudet@umontreal.ca; or to Dr. Santos at the Lipid Clinic Heart Institute, University of São Paulo Medical School Hospital, and Hospital Israelita Albert Einstein, São Paulo 05403-900, Brazil, or at rauldsf@gmail.com.

*A complete list of the HAUSER-RCT investigators is provided in the Supplementary Appendix, available at NEJM.org.

This article was published on August 29, 2020, at NEJM.org.

N Engl J Med 2020;383:1317-27. DOI: $10.1056 /$ NEJMoa2019910 Copyright (c) 2020 Massachusetts Medical Society. 
$\mathrm{F}$ AMILIAL HYPERCHOLESTEROLEMIA IS A genetic condition characterized by an elevated plasma concentration of low-density lipoprotein (LDL) cholesterol starting at birth and an increased risk of premature atherosclerotic cardiovascular disease. ${ }^{1}$ With an estimated prevalence of 1 in 250 in the general population, heterozygous familial hypercholesterolemia is the most frequent monogenic disorder and a major heritable cause of atherosclerotic cardiovascular disease worldwide. ${ }^{2}$

Familial hypercholesterolemia is caused by variants in genes encoding proteins involved in the clearance of LDL particles. More than $90 \%$ of the patients with genetically confirmed familial hypercholesterolemia have mutations in the gene encoding the LDL receptor (LDLR). Familial hypercholesterolemia is also caused by mutations in genes encoding apolipoprotein B (APOB), proprotein convertase subtilisin-kexin type 9 (PCSK9), or LDL receptor adaptor protein 1 (LDLRAP1). ${ }^{1}$ Naturally occurring loss-of-function PCSK9 variants are associated with low LDL cholesterol levels starting at birth and a decreased incidence of atherosclerotic cardiovascular disease in adulthood. ${ }^{3}$ Growing evidence suggests that the pathophysiological atherosclerotic changes begin early in life in patients with familial hypercholesterolemia and that reduction of LDL cholesterol levels in childhood may be important to prevent underlying atherosclerotic cardiovascular disease. ${ }^{4}$ Statins are the standard preferred therapy for the pharmacologic treatment of familial hypercholesterolemia in pediatric patients. Ezetimibe may be added, and current guidelines recommend treatment initiation at the age of 8 or 10 years in heterozygous familial hypercholesterolemia. ${ }^{4,5}$ Despite appropriate treatment, guideline-recommended LDL cholesterol levels are not achieved in some patients with familial hypercholesterolemia. $^{5-7}$

Evolocumab is a fully human monoclonal antibody directed against PCSK9. It prevents PCSK9 from degrading LDL receptor, which increases the availability of LDL receptor and thus reduces LDL cholesterol levels up to $60 \%$ in adults. ${ }^{8}$ Evolocumab was initially approved in 2015 to reduce LDL cholesterol levels in adults with hyperlipidemia, including familial hypercholesterolemia, and in 2017 to reduce the risk of myocardial infarction, stroke, and revascularization in adults with atherosclerotic cardiovascular disease. ${ }^{9-12}$
Here, we describe the results of HAUSER-RCT, a randomized, placebo-controlled, parallel-group, phase 3 trial of evolocumab. The trial was designed to assess the efficacy and safety of evolocumab as an add-on therapy to appropriate statin treatment, with or without ezetimibe, in pediatric patients with familial hypercholesterolemia in whom LDL cholesterol goals had not been achieved.

METHODS

\section{TRIAL DESIGN AND OVERSIGHT}

We conducted the trial from March 2016 through November 2019. The first patient was enrolled on March 24, 2016, and the last on May 30, 2019; the last visit with a trial patient was on November 25 , 2019. The date of the database lock was February 25, 2020. Patients underwent screening and randomization at 47 sites across 23 countries in North America, Latin America, Europe, and the Asia-Pacific region. The trial consisted of a screening period with a maximum duration of 8 weeks followed by a 24-week double-blind treatment phase. Diagnosis of heterozygous familial hypercholesterolemia was made by means of genetic testing or according to applicable clinical diagnostic criteria for heterozygous familial hypercholesterolemia (criteria outlined by the Simon Broome Register Group, the Dutch Lipid Clinic Network, and the Make Early Diagnosis to Prevent Early Deaths [MEDPED] program). Biomarker measurements and pharmacogenetic testing were performed after written informed consent to participate in the trial had been obtained (from the patient or a legal guardian) in accordance with local regulations. An open-label extension study (HAUSER-OLE) is currently ongoing and was offered to patients who completed the present trial.

Amgen sponsored and designed the trialli; the protocol and statistical analysis plan are available with the full text of this article at NEJM.org. The trial protocol and informed-consent form were reviewed and approved by independent ethics committees or institutional review boards at each trial site. Trial activities and oversight were conducted in accordance with the principles of the Declaration of Helsinki, the Good Clinical Practice guidelines of the International Conference on Harmonisation, and relevant regulatory requirements. An independent data monitoring committee and the independent biostatistical group oversaw efficacy and safety data 
on a regular basis. The first draft of the manuscript was written by an Amgen medical writer, in collaboration with and under the direction of the first, second, eighth, and last authors; all the coauthors interpreted the results and contributed to the development of subsequent manuscript drafts and vouch for the completeness and accuracy of the data and for the fidelity of the trial to the protocol.

\section{PATIENTS AND RANDOMIZATION}

Detailed information on patient eligibility criteria was reported previously ${ }^{13}$ (Table S1 in the Supplementary Appendix, available at NEJM.org). Patients 10 to 17 years of age with heterozygous familial hypercholesterolemia who were following a low-fat diet, who had received stable lipidlowering therapy for at least 4 weeks before screening, and who had an LDL cholesterol level of $130 \mathrm{mg}$ per deciliter (3.4 mmol per liter) or more and a triglyceride level of $400 \mathrm{mg}$ per deciliter (4.5 mmol per liter) or less were screened. Patients were randomly assigned in a 2:1 ratio to receive monthly subcutaneous injections of either evolocumab $(420 \mathrm{mg})$ or placebo for 24 weeks. Randomization was stratified according to the LDL cholesterol level at the time of screening ( $<160$ vs. $\geq 160 \mathrm{mg}$ per deciliter $[4.1 \mathrm{mmol}$ per liter]) and the age at baseline ( $<14$ vs. $\geq 14$ years).

\section{END POINTS}

The primary efficacy end point was the percent change in LDL cholesterol level from baseline to week 24 . Secondary end points included the mean percent change in LDL cholesterol level from baseline to weeks 22 and 24, the absolute change in LDL cholesterol level from baseline to week 24, and the percent changes in non-high-density lipoprotein (HDL) cholesterol level, apolipoprotein B level, the ratio of total cholesterol to HDL cholesterol, and the ratio of apolipoprotein $\mathrm{B}$ to apolipoprotein A1 from baseline to week 24. Details regarding other tertiary and exploratory end points are provided in the Supplementary Appendix.

\section{SAFETY}

Safety analyses included the incidence of adverse events that occurred during the treatment period. Laboratory assessments included levels of hormones and vitamins $\mathrm{A}, \mathrm{D}, \mathrm{E}$, and $\mathrm{K}$ and the development of anti-evolocumab antibodies. Specific safety tests were conducted at baseline and week
24 to assess cognitive function (Cogstate battery of cognitive tests), pubertal growth and development (Tanner staging), carotid intima-media thickness for atherosclerosis progression, ${ }^{13}$ and changes on electrocardiography (ECG).

\section{STATISTICAL ANALYSIS}

A sample of 150 patients who were randomly assigned in a 2:1 ratio to receive evolocumab or placebo was calculated to be sufficient to generate $99 \%$ power to test the superiority of evolocumab (420 mg) over placebo for the primary end point. The sample size was calculated with the use of a two-sided t-test with a type I error rate of 0.05 and the following assumptions: a $40 \%$ lower level of LDL cholesterol with evolocumab than with placebo, a common standard deviation of $20 \%$, and $20 \%$ of patients discontinuing the assigned regimen before trial completion (Fig. S1). Efficacy and safety analyses included all randomly assigned patients who received at least one dose of evolocumab or placebo. Additional information is provided in the Supplementary Appendix.

\section{RESULTS}

\section{PATIENTS}

From March 2016 through May 2019, a total of 158 patients underwent randomization, of whom 157 (99\%) received either evolocumab (104 patients) or placebo (53 patients) (Fig. S2). The demographic and clinical characteristics of the patients at baseline were generally balanced between the two groups (Table 1$)$. The mean $( \pm S D$ ) age of the patients was $13.7 \pm 2.4$ years, 88 patients (56\%) were female, and 133 (85\%) were White. A total of 18 patients (11\%) had two or more risk factors for atherosclerotic cardiovascular disease; 52 (33\%) had a first-degree family history of premature atherosclerotic cardiovascular disease. All the patients received a diagnosis of heterozygous familial hypercholesterolemia at screening. A total of 104 patients $(66 \%)$ received a molecular diagnosis of familial hypercholesterolemia; of these, 100 patients (96\%) had a mutation in LDLR, 3 (3\%) had a mutation in $A P O B$, and $1(1 \%)$ had a mutation in PCSK9. At baseline, 124 patients (79\%) were using high-intensity or moderate-intensity statins, and 21 (13\%) were also taking ezetimibe. No changes in background lipid-lowering therapy were reported at any time during the trial. 


\begin{tabular}{|c|c|c|}
\hline Characteristic & $\begin{array}{c}\text { Evolocumab } \\
(N=104)\end{array}$ & $\begin{array}{l}\text { Placebo } \\
(\mathrm{N}=53)\end{array}$ \\
\hline \multicolumn{3}{|l|}{ Age } \\
\hline Mean - yr & $13.7 \pm 2.3$ & $13.7 \pm 2.5$ \\
\hline \multicolumn{3}{|l|}{ Distribution - no. (\%) } \\
\hline$<14 \mathrm{yr}$ & $48(46)$ & $25(47)$ \\
\hline$\geq 14 \mathrm{yr}$ & $56(54)$ & $28(53)$ \\
\hline Female sex — no. (\%) & $61(59)$ & $27(51)$ \\
\hline White race - no. (\%) $†$ & $89(86)$ & $44(83)$ \\
\hline \multicolumn{3}{|l|}{ Weight — kg } \\
\hline Median (IQR) & $55.3(48.0-68.8)$ & $53.3(42.4-61.0)$ \\
\hline 10th percentile & 35.7 & 35.8 \\
\hline 90th percentile & 79.0 & 80.6 \\
\hline \multicolumn{3}{|l|}{ Height $-\mathrm{cm}$} \\
\hline Median (IQR) & $161.5(152.0-168.0)$ & $158.5(148.0-165.5)$ \\
\hline 10th percentile & 143.8 & 143.0 \\
\hline 90th percentile & 173.0 & 175.2 \\
\hline \multicolumn{3}{|l|}{ BMI-for-age category — no. (\%) } \\
\hline Underweight & $2(2)$ & $2(4)$ \\
\hline Normal & $62(60)$ & $36(68)$ \\
\hline Overweight & $21(20)$ & $8(15)$ \\
\hline Obese & $19(18)$ & $7(13)$ \\
\hline \multicolumn{3}{|l|}{ Geographic region — no. (\%) } \\
\hline North America & $12(12)$ & $10(19)$ \\
\hline Europe & $68(65)$ & $35(66)$ \\
\hline Latin America & $18(17)$ & $8(15)$ \\
\hline Asia-Pacific & $6(6)$ & 0 \\
\hline \multicolumn{3}{|l|}{ Cardiovascular risk factors — no. (\%)』 } \\
\hline Hypertension & $2(2)$ & $3(6)$ \\
\hline Type 2 diabetes mellitus & $1(1)$ & 0 \\
\hline Family history of premature CHD & $31(30)$ & $21(40)$ \\
\hline Low HDL cholesterol & $40(38)$ & $18(34)$ \\
\hline Diagnosis of heterozygous $\mathrm{FH}-$ no. (\%) & $104(100)$ & $53(100)$ \\
\hline \multicolumn{3}{|l|}{ Genetic } \\
\hline LDLR mutation & $70(67)$ & $30(57)$ \\
\hline$A P O B$ mutation & $2(2)$ & $1(2)$ \\
\hline PCSK9 gain-of-function mutation & 0 & $1(2)$ \\
\hline \multicolumn{3}{|l|}{ Clinical } \\
\hline Simon Broome Register Group criteria & $9(9)$ & $7(13)$ \\
\hline Dutch Lipid Clinic Network criteria & $22(21)$ & $10(19)$ \\
\hline MEDPED program criteria & $1(1)$ & $4(8)$ \\
\hline
\end{tabular}




\begin{tabular}{|c|c|c|}
\hline Characteristic & $\begin{array}{c}\text { Evolocumab } \\
(\mathrm{N}=104)\end{array}$ & $\begin{array}{l}\text { Placebo } \\
(\mathrm{N}=53)\end{array}$ \\
\hline Statin use - no. (\%) & $104(100)$ & $52(98)$ \\
\hline High intensity & $19(18)$ & $7(13)$ \\
\hline Moderate intensity & $63(61)$ & $35(66)$ \\
\hline Low intensity or unknown intensity & $22(21)$ & $10(19)$ \\
\hline Ezetimibe - no. (\%) & $13(12)$ & $8(15)$ \\
\hline \multicolumn{3}{|l|}{ Lipid variables $₫$} \\
\hline LDL cholesterol - mg/dl & $185.0 \pm 45.0$ & $183.0 \pm 47.2$ \\
\hline Non-HDL cholesterol — mg/dl & $203.8 \pm 47.3$ & $200.2 \pm 48.2$ \\
\hline Total cholesterol — mg/dl & $250.7 \pm 47.0$ & $247.3 \pm 49.5$ \\
\hline HDL cholesterol — mg/dl & $46.8 \pm 12.0$ & $47.2 \pm 11.9$ \\
\hline Median triglycerides $(\mathrm{IQR})-\mathrm{mg} / \mathrm{dl}$ & $86.8(63.8-117.0)$ & $78.0(62.5-101.0)$ \\
\hline Median lipoprotein(a) (IQR) - nmol/liter & $50.5(18.0-126.5)$ & $34.0(21.0-147.0)$ \\
\hline $\mathrm{PCSK} 9-\mathrm{ng} / \mathrm{ml}$ & $277.2 \pm 92.1$ & $294.2 \pm 101.3$ \\
\hline
\end{tabular}

* Plus-minus values are means \pm SD. Patients were randomly assigned in a 2:1 ratio to receive monthly subcutaneous injections of either evolocumab $(420 \mathrm{mg})$ or placebo for 24 weeks. Data from all 157 patients were used for analysis. To convert the values for LDL cholesterol to millimoles per liter, multiply by 0.02586 . To convert the values for triglycerides to millimoles per liter, multiply by 0.01129 . Percentages may not total 100 because of rounding. CHD denotes coronary heart disease, FH familial hypercholesterolemia, HDL high-density lipoprotein, IQR interquartile range, LDL low-density lipoprotein, and MEDPED Make Early Diagnosis to Prevent Early Deaths.

$\dagger$ Race was reported by the patient.

7 The body-mass index (BMI) is the weight in kilograms divided by the square of the height in meters. BMI-for-age categories are based on expert committee recommendations ${ }^{14-16}$ (underweight, $<5$ th percentile; normal, $\geq 5$ th percentile to $<85$ th percentile; overweight, $\geq 85$ th percentile to $<95$ th percentile; and obese, $\geq 95$ th percentile).

$\int$ Shown are hypertension and diabetes diagnoses as provided by the investigators and entered in the trial clinical research form; no definition of hypertension was specifically used in the trial. A low HDL cholesterol level was defined as less than $40 \mathrm{mg}$ per deciliter $(1.0 \mathrm{mmol}$ per liter) in patients 10 years of age to younger than 16 years of age, less than $40 \mathrm{mg}$ per deciliter in male patients 16 years of age or older, and less than $50 \mathrm{mg}$ per deciliter ( $1.3 \mathrm{mmol}$ per liter) in female patients 16 years of age or older.

I For lipoprotein(a), 2 patients in the placebo group had missing values; for proprotein convertase subtilisin-kexin type 9 (PCSK9), 7 patients in the evolocumab group and 1 patient in the placebo group had missing values.

\section{EFFICACY}

The mean $( \pm S D)$ level of LDL cholesterol at baseline was $184.3 \pm 45.6 \mathrm{mg}$ per deciliter $(4.8 \pm 1.2$ mmol per liter). Results for the primary and secondary efficacy end points were significantly better with evolocumab than with placebo $(\mathrm{P}<0.001$ for all comparisons, with adjustment for multiple comparisons). At week 24, the mean percent change in LDL cholesterol level was $-44.5 \%$ in the evolocumab group and $-6.2 \%$ in the placebo group, for a difference of -38.3 percentage points (95\% confidence interval [CI], -45.5 to -31.1 ). The mean absolute change in LDL cholesterol level from baseline to week 24 was $-77.5 \mathrm{mg}$ per deciliter ( $-2.0 \mathrm{mmol}$ per liter) in the evolocumab group and $-9.0 \mathrm{mg}$ per deciliter $(-0.2 \mathrm{mmol}$ per liter) in the placebo group, for a difference of $-68.6 \mathrm{mg}$ per deciliter (95\% CI, -83.1 to -54.0 ) $(-1.8 \mathrm{mmol}$ per liter [95\% CI, -2.1 to -1.4$])$ (Table 2).

The efficacy of evolocumab for LDL cholesterol reduction was maintained throughout the trial (Fig. 1). At weeks 22 and 24, the mean percent change from baseline in LDL cholesterol level was $-48.0 \%$ in the evolocumab group and $-5.9 \%$ in the placebo group, for a difference of -42.1 percentage points (Table 2). An LDL cholesterol reduction of more than $50 \%$ at week 22 was achieved in 60 of 97 patients (62\%) in the evolocumab group and 0 of 49 patients in the placebo group. Similarly, at week 24, a total of 43 of 96 patients (45\%) in the evolocumab group and 1 of 44 pa- 


\begin{tabular}{|c|c|c|c|c|}
\hline End Point & $\begin{array}{l}\text { Evolocumab } \\
(\mathrm{N}=104) *\end{array}$ & $\begin{array}{l}\text { Placebo } \\
(\mathrm{N}=53)^{*}\end{array}$ & $\begin{array}{l}\text { Difference } \\
(95 \% \mathrm{Cl}) * \mathrm{i}\end{array}$ & $\begin{array}{l}\text { Adjusted } \\
\text { P Value }\end{array}$ \\
\hline $\begin{array}{l}\text { Percent change in LDL cholesterol from baseline to } \\
\text { wk } 24(95 \% \mathrm{Cl})\end{array}$ & $-44.5(-48.8$ to -40.3$)$ & $-6.2(-12.3$ to -0.2$)$ & $-38.3(-45.5$ to -31.1$)$ & $<0.001$ \\
\hline $\begin{array}{l}\text { Percent change in LDL cholesterol from baseline to } \\
\text { wk } 22 \text { and } 24(95 \% \mathrm{Cl})\end{array}$ & $-48.0(-51.7$ to -44.2$)$ & $-5.9(-11.1$ to -0.6$)$ & $-42.1(-48.3$ to -35.8$)$ & $<0.001$ \\
\hline $\begin{array}{l}\text { Absolute change in LDL cholesterol from baseline to } \\
\text { wk } 24(95 \% \mathrm{Cl})-\mathrm{mg} / \mathrm{dl}\end{array}$ & $-77.5(-86.1$ to -68.9$)$ & $-9.0(-21.1$ to 3.2$)$ & $-68.6(-83.1$ to -54.0$)$ & $<0.001$ \\
\hline $\begin{array}{l}\text { Percent change in non-HDL cholesterol from base- } \\
\text { line to wk } 24(95 \% \mathrm{Cl})\end{array}$ & $-41.2(-45.2$ to -37.2$)$ & $-6.1(-11.8$ to -0.5$)$ & $-35.0(-41.8$ to -28.3$)$ & $<0.001$ \\
\hline $\begin{array}{l}\text { Percent change in apolipoprotein B from baseline to } \\
\text { wk } 24(95 \% \mathrm{Cl})\end{array}$ & $-34.9(-38.6$ to -31.1$)$ & $-2.4(-7.7$ to 3.0$)$ & $-32.5(-38.8$ to -26.1$)$ & $<0.001$ \\
\hline $\begin{array}{l}\text { Percent change in ratio of total cholesterol to HDL } \\
\text { cholesterol from baseline to wk } 24(95 \% \mathrm{Cl})\end{array}$ & $-35.0(-38.6$ to -31.4$)$ & $-4.7(-9.8$ to 0.5$)$ & $-30.3(-36.4$ to -24.2$)$ & $<0.001$ \\
\hline $\begin{array}{l}\text { Percent change in ratio of apolipoprotein } \mathrm{B} \text { to apoli- } \\
\text { poprotein Al from baseline to wk } 24(95 \% \mathrm{Cl})\end{array}$ & $-37.0(-40.9$ to -33.2$)$ & $-0.6(-6.2$ to 4.9$)$ & $-36.4(-43.0$ to -29.8$)$ & \\
\hline $\begin{array}{l}\text { Percent change in lipoprotein (a) from baseline to wk } \\
\qquad 24(95 \% \mathrm{Cl}) \div\end{array}$ & $-7.4(-20.1$ to 5.3$)$ & 1.7 (-17.0 to 20.5$)$ & & \\
\hline $\begin{array}{l}\text { Percent change in lipoprotein (a) from baseline to wk } \\
22 \text { and } 24(95 \% \mathrm{CI}) \div\end{array}$ & $-10.3(-18.7$ to -1.9$)$ & $1.6(-10.4$ to 13.7$)$ & & \\
\hline
\end{tabular}

* Shown are least-squares means from the repeated-measures model, which includes trial group, stratification factor of age at baseline and LDL cholesterol level at the time of screening (from the interactive voice-response system), scheduled visit, and the interaction of treatment with scheduled visit as covariates.

$\uparrow$ The difference is the value in the evolocumab group minus the value in the placebo group. Differences are expressed as percentage points, except for the absolute change in LDL cholesterol level (expressed as milligrams per deciliter).

$\checkmark$ Lipoprotein (a) was a tertiary end point.

tients $(2 \%)$ in the placebo group had an LDL cholesterol reduction of more than $50 \%$ (Table S2). An LDL cholesterol level of less than $130 \mathrm{mg}$ per deciliter at week 22 was achieved in 77 of 97 patients (79\%) in the evolocumab group and 7 of 49 patients $(14 \%)$ in the placebo group (Table S2). At week 24, an LDL cholesterol level of less than $130 \mathrm{mg}$ per deciliter was achieved in 71 of 96 patients (74\%) in the evolocumab group and 10 of 44 patients (23\%) in the placebo group (Table S2).

Evolocumab showed a benefit with respect to other secondary lipid-related end points, including non-HDL cholesterol and apolipoprotein B (Table 2). Results of sensitivity analyses of the primary and secondary end points, including an analysis in which assessments were made outside the prespecified time window of week 24 (Table S3), were consistent with the results of the primary analysis (Table S3). The primary endpoint analysis at the patient level showed a consistently greater reduction in LDL cholesterol levels with evolocumab than with placebo (Fig. S3).

Evolocumab showed a benefit with respect to the primary and secondary efficacy end points in additional subgroup analyses (Fig. 2). The effect of evolocumab on LDL cholesterol lowering was similar in patients younger than 14 years of age and those 14 years of age or older (Fig. 2). Furthermore, the mean percent decrease in the lipoprotein(a) level at week 24 and at weeks 22 and 24 was numerically greater with evolocumab than with placebo (Table 2). Evolocumab treatment resulted in a reduction in the serum levels of circulating unbound PCSK9, with maximal suppression of PCSK9 and LDL cholesterol at week 22 correlating with a peak concentration of evolocumab (Fig. S4).

\section{SAFETY}

The incidence of adverse events that occurred during the treatment period was similar in the evolocumab group and the placebo group. A total of 64 patients (62\%) in the evolocumab group and $34(64 \%)$ in the placebo group reported at least one adverse event (Table 3). One patient (1\%) in the evolocumab group reported a serious adverse event of cholelithiasis that was not considered by 


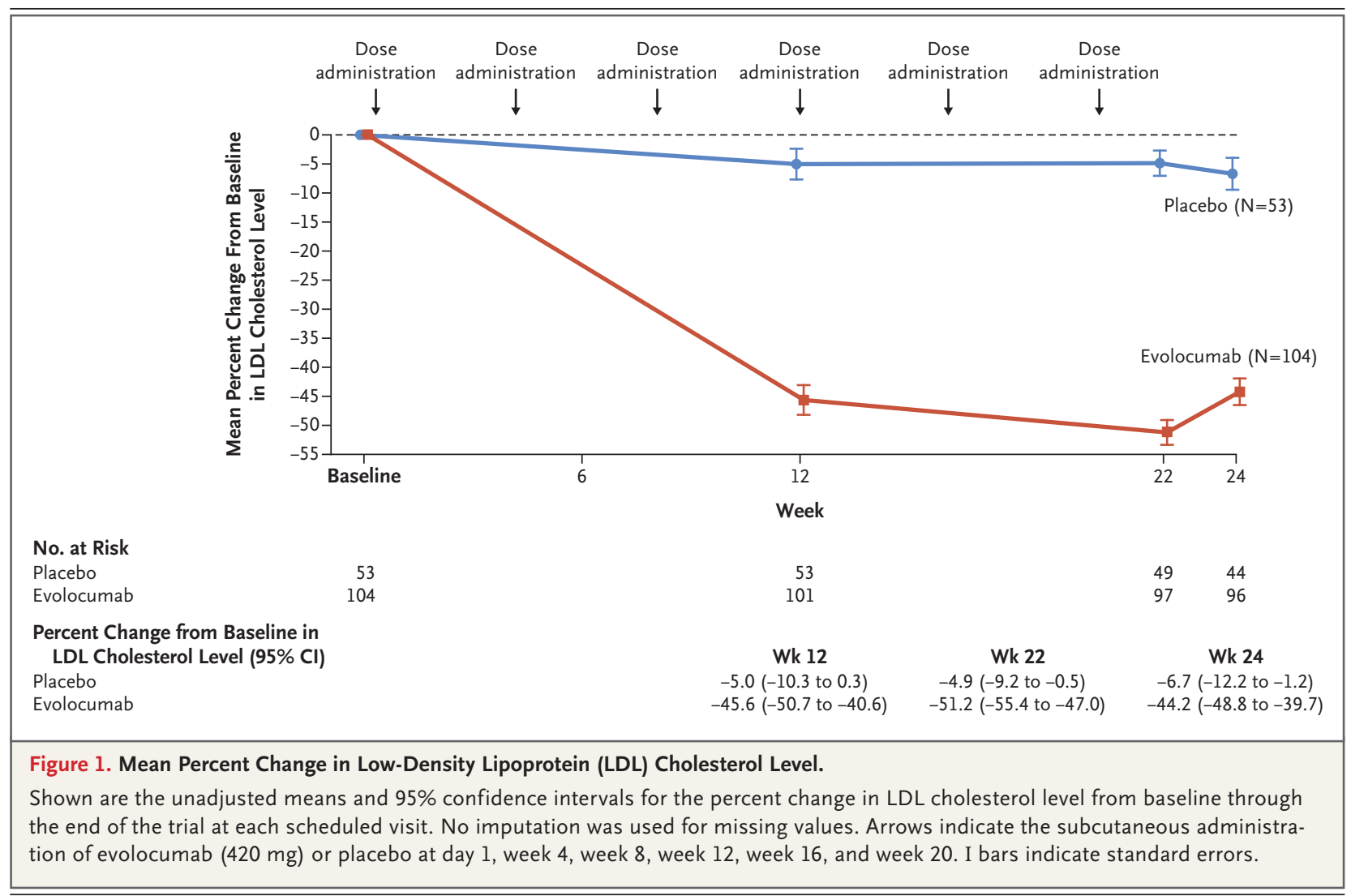

the investigators and data monitoring committee to be related to the drug; another patient in the evolocumab group had a nonserious treatmentrelated adverse event of arthropathy that led to discontinuation of the drug (Table 3). The most common adverse events $(>2 \%$ of the patients in either group) with a higher incidence (by $>1$ percentage point) in the evolocumab group than in the placebo group were headache, oropharyngeal pain, influenza, influenza-type illness, upper respiratory tract infection, and constipation. In the evolocumab group, an injection-site reaction occurred in 1 patient (1\%), as compared with no patients in the placebo group (Table 3). Four patients in the evolocumab group (4\%) discontinued treatment with the drug: 1 had a nonserious treatment-related adverse event of arthropathy (mentioned above), 2 requested discontinuation, and 1 missed a scheduled visit at which the final dose would have been administered.

\section{ADDITIONAL EFFICACY AND SAFETY ASSESSMENTS}

At week 24, mean changes from baseline in pubertal development (Tanner stage for male pa- tients and female patients [Table S4]) and growth variables (height, weight, and body-mass index [Table S9]) were similar in the evolocumab and placebo groups. Results for laboratory variables (including levels of steroid hormones, fat-soluble vitamins [vitamins A, D, E, and K], glucose, and glycated hemoglobin) were similar in the two groups at week 24 (Table S5). Changes from baseline in carotid intima-media thickness (Table S6), neurologic assessment with the Cogstate battery of cognitive tests (Table S7), and ECG measures (Table S8) at week 24 were similar in the evolocumab and placebo groups. Anti-evolocumab antibodies developed in no patients during the trial, and no onset of diabetes was observed (Table 3).

\section{DISCUSSION}

In this multicenter, randomized, controlled trial involving pediatric patients with heterozygous familial hypercholesterolemia, the PCSK9 inhibitor evolocumab, when added to statin therapy with or without ezetimibe, was associated with a 


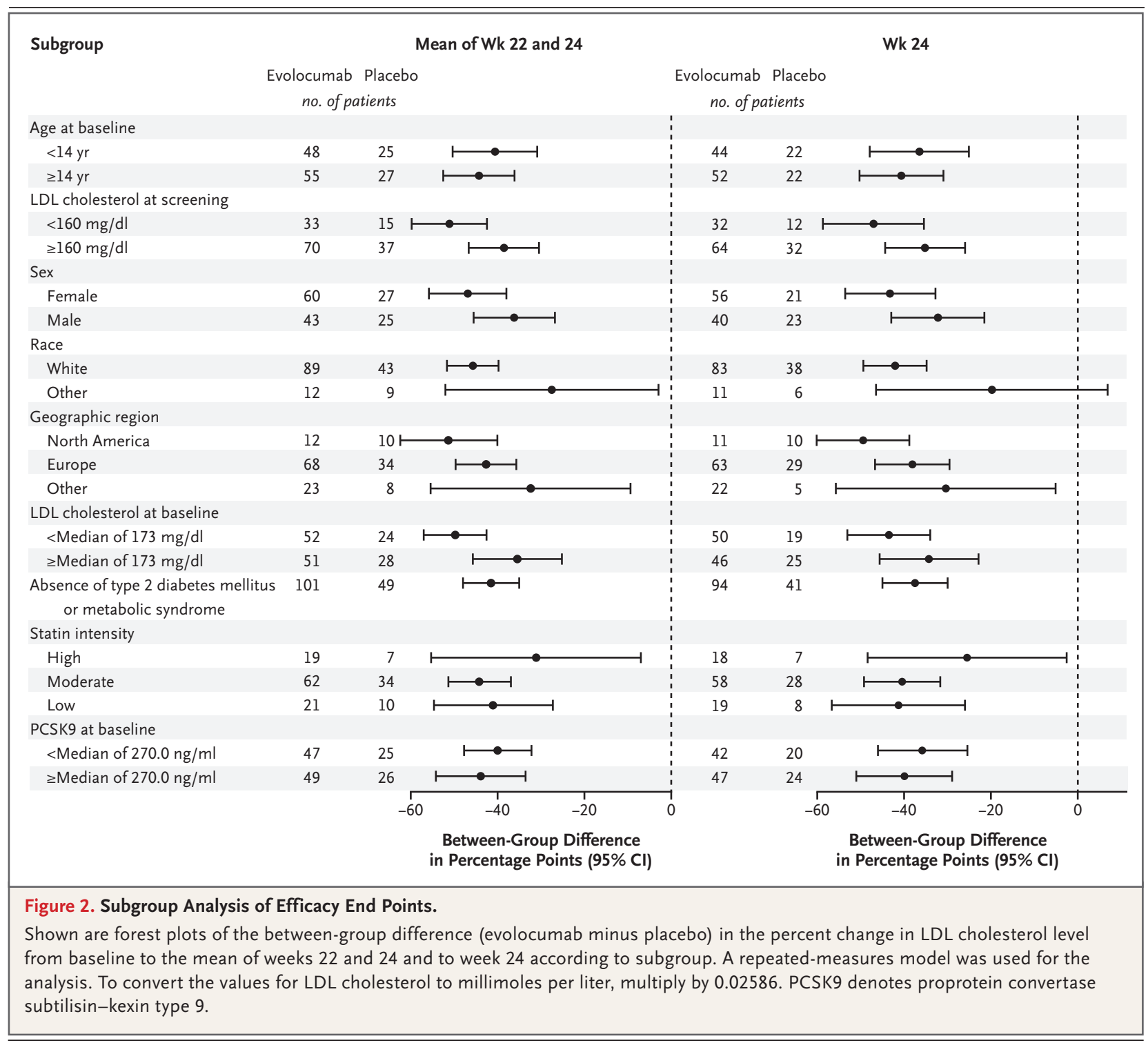

significantly greater reduction in the LDL cholesterol level from baseline to week 24 than placebo (difference, -38.3 percentage points or $-68.6 \mathrm{mg}$ per deciliter). At weeks 22 and 24, the between-group difference in the mean percent change from baseline in LDL cholesterol level was -42.1 percentage points. Significant benefits with respect to other lipid variables in this pediatric population were also observed. No safety concerns emerged from the analyses of the safety data.

Statins, ezetimibe, and bile acid-binding resins are currently approved therapies to lower LDL cholesterol levels in pediatric patients with heterozygous familial hypercholesterolemia. De- spite these medications, a recent European study showed that guideline-recommended LDL cholesterol goals of less than $130 \mathrm{mg}$ per deciliter, more modest than those for adults, ${ }^{1}$ were not achieved in 41 to $56 \%$ of these pediatric patients in most countries, although treatment may not have been appropriate in all the patients studied. ${ }^{7}$ In the present trial, 124 patients (79\%) were receiving high-intensity or moderate-intensity statins, and only a minority (21 patients [13\%]) were receiving ezetimibe. Background lipid-lowering treatment reflected the diversity of the real-life management of pediatric familial hypercholesterolemia in the 23 countries from which the participants originated. 
Statins are currently the first-line treatment for pediatric patients who may need pharmacologic therapy. Results of a recent study suggest that early initiation of statin treatment reduces subclinical atherosclerosis progression (as measured by carotid intima-media thickness), which is likely to result in a decrease in clinical atherosclerotic cardiovascular disease events at later stages of life. ${ }^{17}$ Despite the fact that the recommended statin dose in the pediatric population is lower than in adults, the care of the majority of pediatric patients with familial hypercholesterolemia will be adequately managed with statins with or without ezetimibe and will not involve additional treatment. However, LDL cholesterol goals are not attained in some patients owing to limited drug response, side effects, or poor treatment adherence. The results of the present trial suggest that if an additional therapy is indicated, evolocumab might be considered. Newer potential treatments such as bempedoic acid, ${ }^{18}$ gemcabene, ${ }^{19}$ small interfering RNA against PCSK9 (inclisiran), ${ }^{20}$ and the monoclonal antibody against angiopoietin-like 3 inhibitor (evinacumab) ${ }^{21}$ have not yet been evaluated in pediatric patients with heterozygous familial hypercholesterolemia.

A few studies have examined the efficacy and safety of PCSK9 inhibitors in a limited number of pediatric patients with heterozygous familial hypercholesterolemia. The Trial Evaluating PCSK9 Antibody in Subjects with LDL Receptor Abnormalities (TESLA) ${ }^{22}$ and the Trial Assessing Long Term Use of PCSK9 Inhibition in Subjects with Genetic LDL Disorders (TAUSSIG) ${ }^{10}$ included a total of 15 pediatric patients, all of whom had homozygous familial hypercholesterolemia. In this small group of patients, the safety profile of evolocumab was similar to that of placebo, and results for LDL cholesterol levels and other lipid variables were better with evolocumab. The ODYSSEY KIDS study, a phase 2, open-label, dose-finding study involving 42 pediatric patients with heterozygous familial hypercholesterolemia, showed a significant reduction in LDL cholesterol levels from baseline to week 8 with alirocumab. ${ }^{23}$

Throughout the present 6-month trial, 153 of 157 patients $(97 \%)$ received all planned doses of evolocumab or placebo, and among the 104 who received evolocumab, binding or neutralizing antibodies developed in no patients. No trends indicative of clinically important treatment-re-

\begin{tabular}{|c|c|c|}
\hline \multicolumn{3}{|l|}{ Table 3. Safety Data.* } \\
\hline Event & $\begin{array}{l}\text { Evolocumab } \\
\quad(N=104)\end{array}$ & $\begin{array}{l}\text { Placebo } \\
(\mathrm{N}=53)\end{array}$ \\
\hline & \multicolumn{2}{|c|}{ no. of patients (\%) } \\
\hline Any adverse event & $64(62)$ & $34(64)$ \\
\hline Serious adverse event & $1(1)$ & 0 \\
\hline $\begin{array}{l}\text { Adverse event leading to discontinuation of } \\
\text { regimen }\end{array}$ & $1(1)$ & 0 \\
\hline Serious & 0 & 0 \\
\hline Nonserious & $1(1)$ & 0 \\
\hline \multicolumn{3}{|l|}{ Most common adverse events } \\
\hline Nasopharyngitis & $12(12)$ & $6(11)$ \\
\hline Headache & $11(11)$ & $1(2)$ \\
\hline Oropharyngeal pain & $7(7)$ & 0 \\
\hline Influenza & $6(6)$ & $2(4)$ \\
\hline Upper respiratory tract infection & $6(6)$ & $1(2)$ \\
\hline Gastroenteritis & $5(5)$ & $4(8)$ \\
\hline Pyrexia & $3(3)$ & $3(6)$ \\
\hline Constipation & $3(3)$ & 0 \\
\hline Influenza-like illness & $3(3)$ & 0 \\
\hline Injection-site reaction & $1(1)$ & 0 \\
\hline \multicolumn{3}{|l|}{ Laboratory results at wk 24} \\
\hline Aminotransferase level $>3 \times$ ULN & 0 & 0 \\
\hline Creatine kinase level $>5 \times$ ULN & 0 & 0 \\
\hline Development of anti-evolocumab antibodies $\uparrow$ & 0 & - \\
\hline
\end{tabular}

* ULN denotes the upper limit of the normal range.

$\uparrow$ Anti-evolocumab antibody assessment by means of an electrochemiluminescence-based immunoassay was performed at day 1 (randomization), week 12 , and week 24 (end of the trial). None of the patients tested positive at any time point.

lated laboratory or vital-sign abnormalities, abnormal physical examination findings (including findings on growth and pubertal development), or cognitive changes were observed. Moreover, there was no evidence of clinically relevant effects of evolocumab on steroid hormone biosynthesis or on levels of vitamins (A, D, E, and K). Both the evolocumab and placebo groups received subcutaneous injections every 4 weeks. No patient dropped out owing to adverse events related to injection.

Our results include safety and efficacy data from this randomized, placebo-controlled trial of a PCSK9 inhibitor in pediatric patients with heterozygous familial hypercholesterolemia. The response to and safety of evolocumab at 24 weeks were consistent in all prespecified subgroups. 
However, the present trial has certain limitations. Despite the fact that it was conducted in 23 countries and five continents, there was a preponderance of White patients. The relatively short duration of the trial precluded the assessment of the effects of evolocumab on carotid intima-media thickness, neurocognition, and growth and development. The long-term safety and efficacy of evolocumab in the management of familial hypercholesterolemia in pediatric patients will require further study.

We found that monthly evolocumab provided substantial LDL cholesterol lowering at 24 weeks when added to background lipid-lowering therapy in pediatric patients with heterozygous familial hypercholesterolemia.

\section{Supported by Amgen.}

Dr. Santos reports receiving consulting fees and lecture fees from AstraZeneca, Aché, Libbs, and Novo Nordisk, grant support, consulting fees, and lecture fees from Amgen and Sanofi-Regeneron Pharmaceuticals, lecture fees from EMS, Merck, Merck Sharp \& Dohme, PTC Therapeutics, and Pfizer, fees for coordinating a research protocol from Esperion, grant support from Kowa, and consulting fees from Abbott; Dr. Ruzza, being employed by and owning stock in Amgen and holding pending patent 63/032451 on PCSK9 inhibitors and methods of use thereof to treat cholesterol-related disorders; Dr. Hovingh, receiving grant support, consulting fees, fees for serving on a speakers bureau, and advisory board fees, all paid to his institution, from Aegerion Pharmaceuticals, Amgen, Regeneron Phar- maceuticals, and Sanofi, receiving grant support, paid to his institution, from AstraZeneca, Eli Lilly, Genzyme, Kowa, Pfizer, Roche, the Medicines Company, and Ionis Pharmaceuticals, and being employed by Novo Nordisk; Dr. Kurtz, being employed by and owning stock in Amgen; Dr. Hamer and Mr. Bridges, being employed by and owning stock in Amgen and holding pending patent 63/032451 on PCSK9 inhibitors and methods of use thereof to treat cholesterol-related disorders; Dr. Bergeron, receiving grant support, advisory board fees, and lecture fees from Amgen and Sanofi, grant support from Regeneron Pharmaceuticals, Akcea Therapeutics-Ionis Pharmaceuticals, the Medicines Company, Kowa, Bayer, Novartis, and Novo Nordisk, and advisory board fees from Akcea Therapeutics; Dr. Descamps, receiving grant support, honoraria, and advisory board fees from Amgen, Sanofi, and Merck Sharp \& Dohme and honoraria from Servier, Mylan, Fresenius Medical Care Belgium, and Eurogenerics; Dr. Kastelein, receiving consulting fees from AstraZeneca, CiVi Biopharma, CSL Behring, Draupnir Bio, Esperion, Gemphire Therapeutics, Madrigal Pharmaceuticals, Matinas BioPharma, NorthSea Therapeutics, Novo Nordisk, Novartis, Reneneron Pharmaceuticals, REGENXBIO, Staten Biotechnology, 89bio, Omeicos Therapeutics, and Serometrix; and Dr. Gaudet, receiving grant support from Esperion, Gemphire Therapeutics, Pfizer, and the Medicines Company and grant support and consulting fees from HDL Therapeutics, Regeneron Pharmaceuticals, and Sanofi. No other potential conflict of interest relevant to this article was reported.

Disclosure forms provided by the authors are available with the full text of this article at NEJM.org.

A data sharing statement provided by the authors is available with the full text of this article at NEJM.org.

We thank the patients, their families, and all the investigators involved in this study. Medical writing and editorial assistance with a previous version of the manuscript, under the direction of the authors, was provided by Qais Al-Hadid, an Amgen medical writer.

\section{APPENDIX}

The authors' affiliations are as follows: the Lipid Clinic Heart Institute, University of São Paulo Medical School Hospital and Hospital Israelita Albert Einstein, São Paulo (R.D.S.); Amgen, Thousand Oaks, CA (A.R., C.E.K, A.H.); the Departments of Vascular Medicine (G.K.H., J.J.P.K.) and Pediatrics (A.W., I.L.), Amsterdam UMC, Amsterdam; the Cardiology Department, Geneva University Hospital, Geneva (F.M.); the Biostatistics Department, Amgen, Cambridge (I.B.), and the Department of Clinical Inherited Metabolic Disorders, Birmingham Children's Hospital, Birmingham (S.S.) - both in the United Kingdom; the Rare Diseases and Clinical Genetics Unit, Academic Pediatric Department, Bambino Gesù Children's Hospital (A.B.), and the Department of Molecular Medicine, Umberto I Hospital, Sapienza University of Rome (C.S.), Rome; the Lipid Clinic, Department of Medicine, Centre Hospitalier Universitaire de Québec-Université Laval, Quebec (J.B.), and the Clinical Lipidology and Rare Lipid Disorders Unit, Community Genomic Medicine Centre and ECOGENE-21, Department of Medicine, Université de Montréal, Chicoutimi, QC (D.G.) - both in Canada; the 2nd Department of Pediatrics, Semmelweis University, Budapest, Hungary (T.S.); the Department of Internal Medicine, Centres Hospitaliers Jolimont, La Louvière, Belgium (O.S.D.); and the Division of Pediatric Pulmonology, Allergology, and Endocrinology, Department of Pediatrics and Adolescent Medicine, Medical University of Vienna, Vienna (S.G.-P.).

\section{REFERENCES}

1. Defesche JC, Gidding SS, Harada-Shiba M, Hegele RA, Santos RD, Wierzbicki AS. Familial hypercholesterolaemia. Nat Rev Dis Primers 2017;3:17093.

2. Hu P, Dharmayat KI, Stevens CAT, et al. Prevalence of familial hypercholesterolemia among the general population and patients with atherosclerotic cardiovascular disease: a systematic review and metaanalysis. Circulation 2020;141:1742-59.

3. Cohen JC, Boerwinkle E, Mosley TH Jr, Hobbs HH. Sequence variations in PCSK9, low LDL, and protection against coronary heart disease. N Engl J Med 2006; 354:1264-72

4. Wiegman A, Gidding SS, Watts GF, et al. Familial hypercholesterolaemia in children and adolescents: gaining decades of life by optimizing detection and treatment. Eur Heart J 2015;36:2425-37.

5. Grundy SM, Stone NJ, Bailey AL, et al. 2018 AHA/ACC/AACVPR/AAPA/ABC/ACPM/ ADA/AGS/APhA/ASPC/NLA/PCNA guideline on the management of blood cholesterol: a report of the American College of Cardiology/American Heart Association
Task Force on Clinical Practice Guidelines. Circulation 2019;139(25):e1082-e1143.

6. Mach F, Baigent C, Catapano AL, et al. 2019 ESC/EAS guidelines for the management of dyslipidaemias: lipid modification to reduce cardiovascular risk. Eur Heart J 2020;41:111-88.

7. Ramaswami U, Futema M, Bogsrud $\mathrm{MP}$, et al. Comparison of the characteristics at diagnosis and treatment of children with heterozygous familial hypercholesterolaemia (FH) from eight European countries. Atherosclerosis 2020;292:178-87. 
8. Ito MK, Santos RD. PCSK9 inhibition with monoclonal antibodies: modern management of hypercholesterolemia. J Clin Pharmacol 2017;57:7-32.

9. Sabatine MS, Giugliano RP, Keech AC, et al. Evolocumab and clinical outcomes in patients with cardiovascular disease. N Engl J Med 2017;376:1713-22.

10. Santos RD, Stein EA, Hovingh GK, et al. Long-term evolocumab in patients with familial hypercholesterolemia. J Am Coll Cardiol 2020;75:565-74.

11. Raal FJ, Stein EA, Dufour R, et al. PCSK9 inhibition with evolocumab (AMG 145 ) in heterozygous familial hypercholesterolaemia (RUTHERFORD-2): a randomised, double-blind, placebo-controlled trial. Lancet 2015;385:331-40.

12. Koren MJ, Sabatine MS, Giugliano RP, et al. Long-term efficacy and safety of evolocumab in patients with hypercholesterolemia. J Am Coll Cardiol 2019;74:2132 46.

13. Gaudet D, Langslet G, Gidding SS et al. Efficacy, safety, and tolerability of evolocumab in pediatric patients with het erozygous familial hypercholesterolemia: rationale and design of the HAUSER-RCT study. J Clin Lipidol 2018;12:1199-207.

14. Barlow SE, Expert Committee. Expert Committee recommendations regarding the prevention, assessment, and treatment of child and adolescent overweight and obesity: summary report. Pediatrics 2007; 120:Suppl 4:S164-S192.

15. Cote AT, Harris KC, Panagiotopoulos C, Sandor GG, Devlin AM. Childhood obesity and cardiovascular dysfunction. J Am Coll Cardiol 2013;62:1309-19.

16. Whitlock EP, Williams SB, Gold R, Smith PR, Shipman SA. Screening and interventions for childhood overweight: a summary of evidence for the US Preventive Services Task Force. Pediatrics 2005; 116(1):e125-e144.

17. Luirink IK, Wiegman A, Kusters DM, et al. 20-Year follow-up of statins in children with familial hypercholesterolemia. N Engl J Med 2019;381:1547-56.

18. Goldberg AC, Leiter LA, Stroes ESG, et al. Effect of bempedoic acid vs placebo added to maximally tolerated statins on low-density lipoprotein cholesterol in patients at high risk for cardiovascular dis- ease: the CLEAR Wisdom randomized clinical trial. JAMA 2019;322:1780-8.

19. Gaudet D, Durst R, Lepor N, et al. Usefulness of gemcabene in homozygous familial hypercholesterolemia (from COBALT-1). Am J Cardiol 2019;124:1876-80. 20. Raal FJ, Kallend D, Ray KK, et al. Inclisiran for the treatment of heterozygous familial hypercholesterolemia. N Engl J Med 2020;382:1520-30.

21. Gaudet D, Gipe DA, Pordy R, et al ANGPTL3 inhibition in homozygous familial hypercholesterolemia. N Engl J Med 2017;377:296-7.

22. Raal FJ, Honarpour N, Blom DJ, et al. Inhibition of PCSK9 with evolocumab in homozygous familial hypercholesterolaemia (TESLA Part B): a randomised, doubleblind, placebo-controlled trial. Lancet 2015; 385:341-50.

23. Daniels S, Caprio S, Chaudhari U, et al. PCSK9 inhibition with alirocumab in pediatric patients with heterozygous familial hypercholesterolemia: the ODYSSEY KIDS study. J Clin Lipidol 2020;14:322330.e5.

Copyright @ 2020 Massachusetts Medical Society.

CLINICAL TRIAL REGISTRATION

The Journal requires investigators to register their clinical trials in a public trials registry. The members of the International Committee of Medical Journal Editors (ICMJE) will consider most reports of clinical trials for publication only if the trials have been registered.

Current information on requirements and appropriate registries is available at www.icmje.org/about-icmje/faqs/. 